\title{
The first suspected case of Corona-virus in the mole of hydatidiform in southern Sistan-Baluchestan, Iran.
}

\author{
Saede Rigi ${ }^{1}$, Roksana Janghorban ${ }^{2}$, Fatemeh Azarkish ${ }^{1}$, Mohammadmehran Aminifard ${ }^{1}$, \\ Shirin bozorgzadeh ${ }^{1}$, mahya zahirnia ${ }^{1}$, mansooreh sadat golmohammadi ${ }^{1}$, Elahe mirtalaie \\ ${ }^{1}$, Arezoo Pirak ${ }^{1}$, and Zahra Atarodi Kashani ${ }^{1}$ \\ ${ }^{1}$ Iranshahr University of Medical Sciences \\ ${ }^{2}$ Shiraz University of Medical Sciences
}

May 28, 2020

\begin{abstract}
The first suspect coronavirus in hydatidiform molar pregnancy accentuates the importance of taking into account the symptoms of Coronavirus in pregnancy, collaboration among physicians of various specialties, the role of convalescence home in continuing outpatient treatment with the aim to prevent the spread of the virus in the family environment.
\end{abstract}

\section{Key Clinical Message}

Outbreak of Coronavirus has affected over throughout the world. This explains the treatment of molar hydatiderma suspected of coronavirus at the beginning of corona epidemic in Sistan and Baluchestan, Iranshahr. Understanding of the management suspected case of Corona-virus in the mole of hydatidiform is considerably important especially in pandemic.

\section{Introduction}

Coronavirus-induced respiratory diseases in human first reported in Wuhan, China on December 31, 2019 quickly broke out in other parts of China and other countries $(1,2)$. The entire human population is at risk for contracting coronavirus disease (COVID-19)(3). Given the immune and anatomical changes during pregnancy, pregnant women are more vulnerable to the virus. The COVID-19 pandemic can therefore cause serious consequences for pregnant women (4-7). The clinical features and effects of COVID-19 during pregnancy and the prenatal outcomes of COVID-19 patients have rarely been addressed in literature (8). Moreover, COVID-19 associated infections have never been reported in molar pregnancy (9). Clinical experience with managing pregnant women and newborns with COVID-19 is also limited in China(10).

\section{Case presentation}

On March 1, 2020, at 5:30 p.m., a 17-year-old primiparous woman with a gestational age of 14 weeks presented with unilateral leg swelling and sudden abdominal distension beginning in the night before to a health center in Sarbaz, Sistan-Baluchestan, Iran. The patient had a history of fever, abdominal pain, dry coughs and shortness of breath during the previous week and nausea, vomiting and fatigue during the previous two months and no family history of respiratory disorders. As a housewife who had not recently traveled, she did not report a history of hypertension, surgery, allergies and underlying diseases.

In the abdominal examination of the patient by a healthcare provider, the baby's heartbeat was not heard and a mismatch was observed between gestational age and fundal height, which corresponded to approximately 24 weeks of gestation. Her physical examinations also showed a body temperature of $37.5 @ \mathrm{C}$, a blood pressure 
of $140 / 90 \mathrm{mmHg}$, a heart rate of $119 \mathrm{bpm}$, a respiratory rate of 18 per minute and oxygen saturation of $97 \%$ at room temperature using an oximeter pulse. Moreover, the experiments showed $\mathrm{HB}=8.4$ and urinary protein of +1 . Given the blood pressure of $140 / 90 \mathrm{mmHg}$, four $\mathrm{g}$ of a $20 \% \mathrm{MgSo} 4.7 \mathrm{H}_{2} \mathrm{O}$ USP solution added to $100 \mathrm{ml}$ of Ringer's solution was intravenously injected within 20 minutes and $10 \mathrm{~g}$ of a $50 \%$ magnesium sulfate solution was injected deep into muscles, $5 \mathrm{~g}$ at each buttock.( Table 1) After receiving a Foley catheter, the patient was transferred in an ambulance to Iran Hospital, Iranshahr, Sistan-Baluchestan as a referral center for pregnant women while receiving $6-8 \mathrm{l} / \mathrm{min}$ of oxygen in a left lateral recumbent position aided by a midwife.

At 9 p.m., the patient was admitted to the maternity ward. She was conscious and pale with hematuria, uterine contractions and vital signs of blood pressure $=110 / 70 \mathrm{mmHg}$, pulse rate $=99$, respiratory rate $=18$, body temperature $=37.3{ }^{\circ} \mathrm{C}$ and urine output $=300 \mathrm{ml} / \mathrm{hr}$. After inserting two IV lines into the left brachial vein $(n=20)$ and the right radial vein $(n=18)$ and performing the tests, the patient immediately underwent monitoring and was visited by a gynecologist. Vaginal examination showed a closed cervix with no bleeding. Moreover, the patient received $1000 \mathrm{ml}$ of normal saline and underwent an ultrasound by adiologist at 9:30 p.m. Complete molar pregnancy was diagnosed with an enlarged heterogeneous uterus $180 \times 90 \mathrm{~cm}$ in size and containing $170 \times 80 \mathrm{~mm}$ cysts. Afterwards, $400 \mu \mathrm{g}$ of vaginal misoprostol (Cytotec) was administered. At 22:10, one unit of packed red blood cells ( $\mathrm{PRBCs}$ ) was administered due to $\mathrm{HB}=5$, and the patient did not show any allergies or shortness of breath during the blood transfusion. Two units of PRBCs were also reserved for the following morning. Counseling was performed by an internist, a cardiologist and infectious disease specialist. The internist evaluated the patient for thyroid storm and signs of pulmonary thromboembolism. Stat anti-coagulant, hydration and hydrocortisone injection (stat and TDS) were administered. Thyroid tests, including TSH:0.2, T4:23 and T3:5.6, were also performed. No heart problems were observed in the patient during the cardiac counseling. The infectious disease specialist suggested the risk of lung metastasis due to molar pregnancy.

The patient was transferred for isolation to the corona ward at 22:50 given her risk of developing COVID19.The treatment began with vancomycin AMP $1 \mathrm{~g}$ BID, hydrocortisone AMP $100 \mathrm{mg} / \mathrm{ml}$ STAT \& TDS, oseltamivir CAP $75 \mathrm{mg}$ for 5 days, kaletra CAP $200 \mathrm{mg}$ for 5 Days and meropenem AMP $1000 \mathrm{mg}$ TDS. The patient's body temperature and oxygen saturation respectively reached $37.4 @ \mathrm{C}$ at $98 \%$ an hour later.

The patient was provided with a surgical mask. All the healthcare team members were also provided with appropriate personal protective gear, and droplet and contact precautions were observed. The patient underwent monitoring at 00:00 a.m. due to dry coughs and respiratory distress. Her oxygen saturation reached $98 \%$ after undergoing oxygen therapy. The RT-PCR sample was taken using oral and throat swabs. The patient was found to be negative for COVID-19 based on the results of the RT-PCR. At 00:10 a.m., 1/3-2/3 IV fluid therapy started at 30 drops/min. At 4:30 a.m., the patient's urine output was below 100 $\mathrm{ml} / \mathrm{hr}$. The patient was transferred to the operating room the following day (March 2, 2020) at 6 a.m. while receiving the second unit of PRBCs. Suction curettage was performed to evacuate the uterus contents. During the curettage, the patient received 20 units of oxytocin in one liter of normal saline plus $0.2 \mathrm{mg}$ of methylergonovine. Samples of the pregnancy products were transferred for pathological examinations.

The patient received the third unit of PRBCs on the second day at 8 a.m. Chest radiography performed by a radiologist at 8:20 a.m. showed multiple nodules in both lungs. After developing severe respiratory distress at 11:40 a.m., the patient was visited by an anesthesiologist, an infectious disease specialist and a gynecologist and then underwent oxygen therapy at $6-8 \mathrm{l} / \mathrm{min}$ with a mask. The patient was intubated at 13:30 and transferred with a respiratory rate of 52, a body temperature of $37 @ \mathrm{C}$ and a pulse rate of 170 to the ICU (Figure 1). Furthermore, CT scan was performed the following day at 16:00 due to shortness of breath, respiratory distress and lowered oxygen saturation (92\%).

The CT scan showed bilateral ground glass patches (Figure 2), suggesting COVID-19. The patient then underwent hydration with $1500 \mathrm{ml}$ of normal saline at 30 drops per minute and oxygen therapy at 8 liters per minute with a mask. The patient had a fever of 38.50 @C on the third day, i.e. March 3, 2020, at 18:00. The Apotel AMP was injected. Symptoms of the patient began to improve on the fourth day, i.e. March 4, 
2020 (Figure 3).

Feeling fine on the fifth day, the patient was discharged from the hospital with a good general health status. The patient was followed up for two weeks at a private recovery center for patients discharged from the hospital. Given the problem of distance, weekly phone follow-ups were performed for 4 weeks after discharge, and the patient did not report any problems during the phone calls.

\section{Discussion}

A pregnant woman requiring mechanical ventilation was the first case with the clinical symptoms and CT scan of the lungs suggesting infection with coronavirus. Molar pregnancy confirmed in the second trimester increased the risk of developing COVID-19 and complicated her conditions. The patient did not report any underlying diseases. Fever, dry coughs and shortness of breath were observed in the patient as in the case of other patients with COVID-19 reported in the literature $(3,8)$.

Knowledge about the clinical spectrum of infection with COVID-19 is very limited in molar pregnancy (11). Despite negative test RT-PCR sample results, clinical findings and the CT scan of the lungs suggesting COVID-19 as a life-threatening condition do not interfere with the disease management (1). The overall effects of COVID-19 on pregnancy and neonatal outcomes and its long-term prognosis are recommended to be further investigated in future studies. Collecting the information of more COVID-19 patients with molar pregnancy can lay the foundations for developing clinical guidelines aimed at managing future cases.

\section{AUTHOR CONTRIBUTIONS}

All authors have read and approved the manuscript, and en-sure that this is the case. MMH, SB, MZ, MSGM, EMT: involved in patient follow-up and management and final approval of the version to be published.SR, RJG and FA: involved in patient management and writing article, drafted the work, and substantively revised it.AP AND ZAK: involved in patient follow-up and management drafted the work and substantively revised it.

References

1. Liu H, Liu F, Li J, Zhang T, Wang D, Lan W. Clinical and CT imaging features of the COVID-19 pneumonia: Focus on pregnant women and children. Journal of infection. 2020.

2. Liu W, Wang Q, Zhang Q, Chen L, Chen J, Zhang B, et al. Coronavirus disease 2019 (COVID-19) during pregnancy: A case series. 2020.

3. Holshue ML, DeBolt C, Lindquist S, Lofy KH, Wiesman J, Bruce H, et al. First case of 2019 novel coronavirus in the United States. New England Journal of Medicine. 2020.

4. Liang H, Acharya G. Novel corona virus disease (COVID-19) in pregnancy: What clinical recommendations to follow? Acta Obstetricia et Gynecologica Scandinavica. 2020;99(4):439-42.

5. Zu ZY, Jiang MD, Xu PP, Chen W, Ni QQ, Lu GM, et al. Coronavirus disease 2019 (COVID-19): a perspective from China. Radiology. 2020:200490.

6. Mor G, Cardenas I, Abrahams V, Guller S. Inflammation and pregnancy: the role of the immune system at the implantation site. Annals of the New York Academy of Sciences. 2011;1221(1):80.

7. Favre G, Pomar L, Musso D, Baud D. 2019-nCoV epidemic: what about pregnancies? The Lancet. 2020;395(10224):e40.

8. Chen H, Guo J, Wang C, Luo F, Yu X, Zhang W, et al. Clinical characteristics and intrauterine vertical transmission potential of COVID-19 infection in nine pregnant women: a retrospective review of medical records. The Lancet. 2020;395(10226):809-15.

9. Bayefsky MJ, Bartz D, Watson KL. Abortion during the Covid-19 Pandemic-Ensuring Access to an Essential Health Service. New England Journal of Medicine. 2020. 
10. Zhu H, Wang L, Fang C, Peng S, Zhang L, Chang G, et al. Clinical analysis of 10 neonates born to mothers with 2019-nCoV pneumonia. Translational pediatrics. 2020;9(1):51.

11. Iqbal SN, Overcash R, Mokhtari N, Saeed H, Gold S, Auguste T, et al. An uncomplicated delivery in a patient with COVID-19 in the United States. New England Journal of Medicine. 2020.

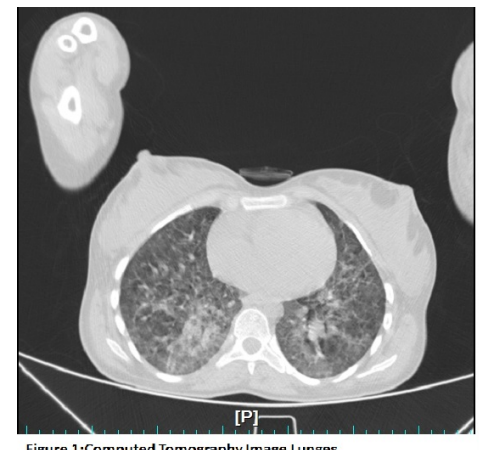

Figure 1:Computed Tomography Image Lunges

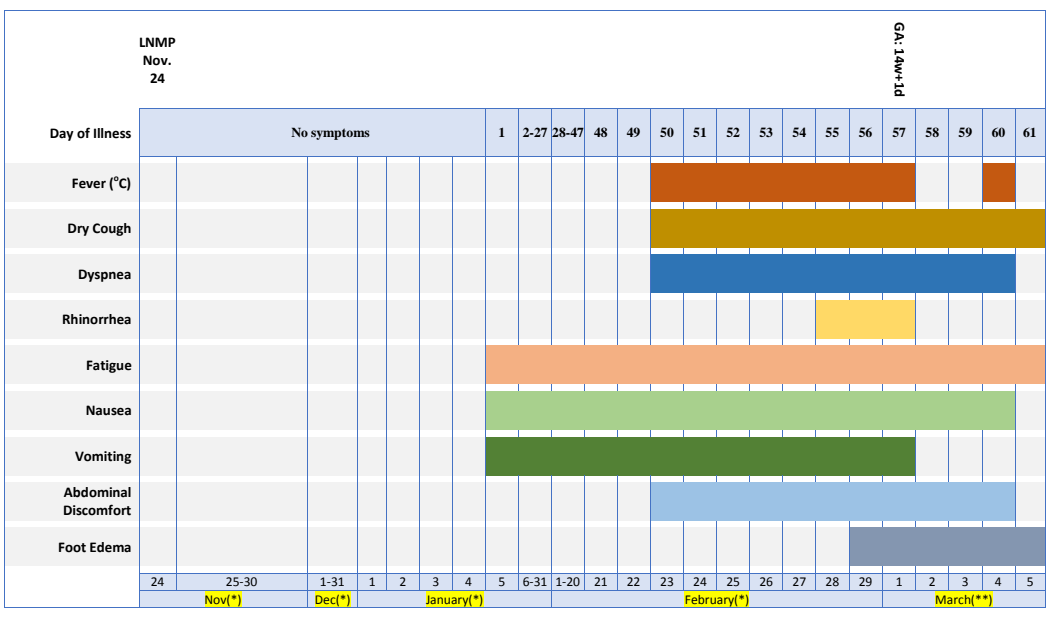

*: Home, **: Hospitalization

Figure 2. Symptoms According to Day of Illness and Day of Hospitalization, March 1 to March 5, 2020. 


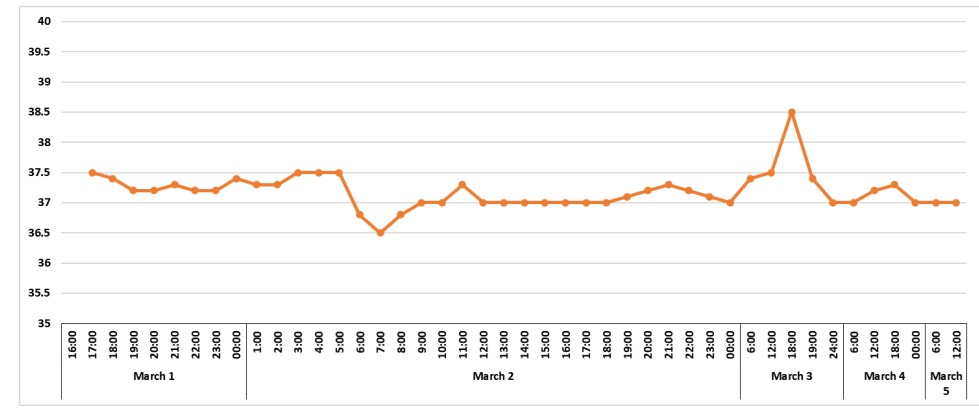

Figure 3. Body temperature recorded during hospitalization, March 1 to March 5, 2020.

\begin{tabular}{|c|c|c|c|c|c|c|}
\hline \multirow{6}{*}{ CBC } & Measure & Reference Range & March1 & March & March 3 & March 4 \\
\hline & WBC (cumm) & 4-10 & 8.5 & 157 & 14.3 & NA. \\
\hline & RBC (Mill/cumm) & 4.2-5.4 & 1.9 & 2.96 & 3.48 & \\
\hline & Hemoglobin $(\mathrm{g} / \mathrm{d}$ ll) & $10-16$ & & & & \\
\hline & Hematocrit (\%) & & 15.3 & 20.7 & 24.3 & N.A. \\
\hline & Platelets (cumm) & $150,000-450,000$ & 182,000 & 107,000 & 115,000 & \\
\hline \multirow{2}{*}{ WBC Differential } & Neut (\%) & & $80 \%$ & $89 \%$ & N.A. & \\
\hline & Lymph(\%) & & & $6 \%$ & N.A. & N.A. \\
\hline \multirow{7}{*}{ Urin Analysis } & Bacteria & & Negative & N.A. & N.A. & N.A. \\
\hline & & $5-7$ & & N.A. & N.A. & N.A. \\
\hline & Specific Gravity & $1.005-1.030$ & 1.030 & N.A. & N.A. & N.A. \\
\hline & Proteins & 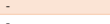 & Trace & N.A. & N.A. & \\
\hline & $\begin{array}{l}\text { Blood } \\
\text { Ketton }\end{array}$ & : & ${ }^{3+}+$ & N.A. & N.A. & N.A. \\
\hline & $\begin{array}{l}\text { Reton } \\
\text { WBC }\end{array}$ & 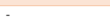 & $2-3$ & $\begin{array}{l}\text { N.A. } \\
\text { N.A. }\end{array}$ & $\begin{array}{l}\text { N.A. } \\
\text { N.A. }\end{array}$ & N.A. \\
\hline & RBC & & Many & $\begin{array}{l}\text { N.A. } \\
\text { N.A. }\end{array}$ & $\begin{array}{l}\text { N.A. } \\
\text { N.A. }\end{array}$ & N.A. \\
\hline \multirow{9}{*}{ Biochemistry } & S.G.O.T (AST) U/L & $<31$ & 47 & 59 & N.A. & $\begin{array}{l}\text { N.A. } \\
\text { N.A. }\end{array}$ \\
\hline & S.G.P.T (ALT) U/L & $<31$ & 60 & 42 & N.A. & N.A. \\
\hline & Phosphatase AlKaline & 64-306 & 36 & 268 & N.A. & N.A. \\
\hline & BUN (mg/dl) & $7-20$ & 11 & 18 & 12 & N.A. \\
\hline & Creatinine (mg/dl) & $0.6-13$ & 0.8 & 0.8 & 0.6 & N.A. \\
\hline & Bilirubin Total (mg/dl) & $0.3-1$ & 0.9 & 3.2 & N.A. & N.A. \\
\hline & Bilirubin Direct & $0.1-0.3$ & 0.3 & 1.9 & N.A. & N.A. \\
\hline & Lactate Dehydrogenase (LDH) U/L & $<480$ & N.A. & 962 & N.A. & N.A. \\
\hline & Creatine Phosphokinase U/L & $24-170$ & N.A. & 840 & N.A. & N.A. \\
\hline \multirow{3}{*}{ Colagulation tests } & PT & $12-14$ & 14 & 13 & N.A. & N.A. \\
\hline & PT & 28 & 28 & 37 & N.A. & N.A. \\
\hline & & & 1.1 & & N.A. & N.A. \\
\hline \multirow{3}{*}{ Hormone } & $\mathrm{TSH}(\mathrm{mll} / \mathrm{L})$ & $0.32-5.2$ & 0.2 & N.A. & N.A. & N.A. \\
\hline & $T 4(\mathrm{mg} / \mathrm{dll})$ & $4.7-12.5$ & 23 & N.A. & N.A. & \\
\hline & $\mathrm{T} 3(\mathrm{ng} / \mathrm{ml})$ & $0.6-2.11$ & 5.6 & N.A. & N.A. & N.A. \\
\hline \multirow{2}{*}{$\begin{array}{l}\text { Immounology \& Serology } \\
\text { Hematology \& Coagulation Test }\end{array}$} & CRP & $<10$ & N.A. & $2+$ & $3+$ & N.A. \\
\hline & ESR (mm/hr) & $15-20$ & N.A. & 46 & N.A. & 57 \\
\hline
\end{tabular}

